\title{
PRODUCTIVIDAD DE LECHUGA Lactuca sativa EN CONDICIONES DE MACROTÚNEL EN SUELO Vitric haplustands
}

\section{PRODUCTIVITY OF LETTUCE Lactuca sativa IN HIGH TUNNEL CONDITIONS ON Vitric haplustands SOIL}

\author{
Pedro Velásquez V. ; Hugo Ruíz E. '; Germán Chaves J. ; Cristina Luna C. ${ }^{4}$
}

Fecha de recepción: Septiembre 19 de 2014 Fecha de aceptación: Noviembre 11 de 2014

\begin{abstract}
RESUMEN
La agricultura urbana hace parte importante en la producción de alimentos para una población cada día más creciente. En este contexto, el objetivo de esta investigación fue evaluar la productividad del cultivo de lechuga Lactuca sativa var. Batavia bajo condiciones de macrotúnel con tres niveles de fertilización. El ensayo se realizó en la localidad de San Felipe, Pasto, Nariño, en un suelo Vitric haplustands; se evaluaron dos tipos de ambientes (condiciones de macrotúnel y a campo abierto), con tres niveles de fertilización: alto115; 69; $210 \mathrm{~kg} / \mathrm{ha}\left(\mathrm{N}, \mathrm{P}_{2} \mathrm{O}_{5}, \mathrm{~K}_{2} \mathrm{O}\right)$, medio $69 ; 23 ; 150 \mathrm{~kg} / \mathrm{ha}\left(\mathrm{N}, \mathrm{P}_{2} \mathrm{O}_{5}, \mathrm{~K}\right)$ y bajo $46 ; 11,5 ; 90 \mathrm{~kg} / \mathrm{ha}\left(\mathrm{N}, \mathrm{P}_{2} \mathrm{O}_{5}, \mathrm{~K}_{2} \mathrm{O}\right)$. El macrotúnel fue construido con arcos de bambú y una cubierta plástica para invernaderos. El ensayo se estableció en un diseño de bloques completos al azar con arreglo de franjas divididas. Las variables agronómicas evaluadas fueron: peso de cabeza (PC), porcentaje de materia seca (aérea y de raíz), diámetro de cabeza (DC), días a cosecha (DCO) y rendimiento (R). Además, se realizó un análisis económico de presupuesto parcial. Los resultados mostraron que el cultivo de lechuga en ambiente de macrotúnel obtuvo en: $P C=2,24 \mathrm{~kg} /$ planta; $\mathrm{DC}=20,33 \mathrm{~cm}$ y $\mathrm{DCO}=74$ días; valores que presentaron diferencias estadísticas significativas con respecto al ambiente campo abierto. A diferencia de lo anterior el porcentaje de materia seca aérea, no presentó diferencias significativas en ninguno de los tratamientos; situación similar aconteció con las dosis de fertilización alto, medio y bajo de $(\mathrm{N}, \mathrm{P}, \mathrm{K})$ y las interacciones de estas, con los dos ambientes evaluados. El análisis económico
\end{abstract}

1 Estudiante de Ingeniería Agronómica. Universidad de Nariño. Facultad de Ciencias Agrícolas, San Juan de Pasto, Colombia. alex_oparin@hotmail.com/

2 Profesor Tiempo completo. I.A., Ph.D. Facultad de Ciencias Agrícolas. Universidad de Nariño. San Juan de Pasto, Colombia. hugoruize@yahoo.com

3 Profesor Hora Cátedra Asociado. I.A., M.Sc. Facultad de Ciencias Agrícolas. Universidad de Nariño. San Juan de Pasto, Colombia. g-ch-j@hotmail.com

4 Profesora Tiempo Completo. I.A., M.Sc. Facultad de Ciencias Agrícolas. Universidad de Nariño. San Juan de Pasto, Colombia. grupopifil@gmail.com 
mostró que las mayores utilidades se obtuvieron en el ambiente de macrotúnel con 11278 pesos/ $\mathrm{m}^{2}$ y una rentabilidad de $507 \%$.

Palabras clave: Fertilización, cubierta plástica, hortalizas.

\begin{abstract}
Urban agriculture is an important part of food production for an increasing population. In this context, the aim of this research was to evaluate lettuce Lactuca sativa var. Batavia crop productivity under high tunnel conditions, with three levels of fertilization. The study was conducted at the locality of San Felipe, in the municipality of Pasto, Nariño, in a Vitric Haplustands soil. Two types of environments (high tunnel condition and open field) were evaluated, with three fertilization levels: high 115; 69; $210 \mathrm{~kg} / \mathrm{ha}\left(\mathrm{N}, \mathrm{P}_{2} \mathrm{O}_{5}, \mathrm{~K} 2 \mathrm{O}\right)$, medium 69; 23; $150 \mathrm{~kg} / \mathrm{ha}\left(\mathrm{N}, \mathrm{P}_{2} \mathrm{O}_{5^{\prime}}\right.$ $\mathrm{K})$, and low 46; 11.5; $90 \mathrm{~kg} / \mathrm{ha}\left(\mathrm{N}, \mathrm{P}_{2} \mathrm{O} 5, \mathrm{~K}_{2} \mathrm{O}\right)$. The high tunnel was built with bamboo arches and a plastic cover for greenhouses. The trial was established in a split plot design under randomized complete blocks with a divided fringe array. The evaluated agronomic variables were: head weight (HW), dry matter percentage (aerial and root), head diameter (HD), days to harvest $(\mathrm{DH})$, and yield (Y). Additionally, an economic partial budget analysis was performed. The results showed that the lettuce cropunder the hightunnel environment exhibited: HW= $2.24 \mathrm{~kg} /$ plant; $\mathrm{HD}=20.33 \mathrm{~cm}$, and $\mathrm{DH}=74$ days. These values showed statistically significant differences compared to the open field. On the other hand, the percentage of aerial dry matter showed no significant differences in any of the treatments. Asimilar situation occurred with high, medium, and low fertilization doses $(\mathrm{N}, \mathrm{P}, \mathrm{K})$, and with the interactions of these with the two environments tested. The economic analysis showed that the highest profits were obtained in thehigh tunnel environment, with 11278 pesos $/ \mathrm{m}^{2}\left(\mathrm{US} \$ 5.13 / \mathrm{m}^{2}\right)$ and a return of $507 \%$.
\end{abstract}

Keywords:fertilization, plastic housing, vegetable.

\section{INTRODUCCIÓN}

La producción de cultivos en los últimos tiempos dada la creciente necesidad de alimentos en el mundo, viene sufriendo procesos de cambios drásticos pasando en algunos cultivos a producirse desde el ámbito urbano, que es donde la agricultura ha tomado gran importancia, por los diferentes sistemas y modalidades de producción que bajo esesistema toma.En ese aspecto Castilla (2007) afirma que el cultivo protegido es un sistema agrícola especializado en el cual se lleva a cabo un cierto control del medio edafoclimático alterando sus condiciones (suelo, temperatura, radiación solar, viento, humedad y composición atmosférica).
El mismo autor, manifiesta que mediante estas técnicas de protección se cultivan plantas, modificando su entorno natural para prolongar el periodo de recolección, alterar los ciclos convencionales, aumentar los rendimientos y mejorar su calidad, estabilizar las producciones y disponer de productos cuando la producción al aire libre se encuentre limitada.

Al respecto Alpi y Tognoni (2010) mencionan que la tendencia que existe desde hace varios años en el campo de los cultivos hortícolas, es hacia la producción anticipada o totalmente fuera de estación (cultivos semiforzados y forzados). Estas instalaciones pueden ser muy diversas y a grandes trazos, una primera clasificación puede 
hacerse entre túneles, cajoneras o semilleros e invernaderos.

En muchas partes del mundo, los túneles altos son hechos de tubos de PVC o tubería galvanizada de espesor variante, éste depende de la durabilidad de la estructura y también de la cantidad de viento que se experimente en la región. En India, los túneles altos son construidos de marcos de bambú fornidos y se cubren con una sola película plástica en la cima y yute en los lados, que excluyen a los insectos y permite un poco de ventilación (Lamont, 2009).

Los macrotúneles, se han convertido en una característica importante de los sistemas intensivos de producción hortícola en varios lugares alrededor del mundo (Jensen, 2000) y pueden mejorar el rendimiento y la calidad de los cultivos por proporcionar condiciones más favorables de crecimiento (Wells, 2000; Wells y Loy, 1993). Al respecto Lamont (2009), señala que la lechuga, es el quinto cultivo que se produce en macrotúneles en todo el mundo.

Dentro de esta modalidad de producción, uno de los aspectos fundamentales a potenciar es la fertilización y nutrición de los cultivos, buscando eficientizar la toma de nutrientes, el mejoramiento de los procesos nutricionales espacial y temporalmente, en los cultivos que se exploten bajo condiciones controladas, debido fundamentalmente a que los procesos tienen una mayor velocidad y eficiencia por los cambios de temperatura, humedad y demás factores edafoclimaticos que están involucrados en los procesos productivos en este aspecto Malavolta (2006), manifiesta que la temperatura es una medida del contenido calorífico del suelo, que influencia en forma directa la germinación de las semillas, la emergencia de los cultivos, el enraizamiento de estacas o el crecimiento de las raíces en general, incrementando en forma sustancial la actividad de los microorganimos, la disponibilidad y absorción de nutrientes desde la solución del suelo, así como los contenidos de agua y en general las reacciones del suelo y la nutrición de la planta.

Los procesos anteriores permiten que los cultivos bajo cubiertas, sean más precoces dada la influencia de la temperatura y humedad que permiten procesos más eficientes en los cultivos que bajo este tipo de macrotuneles se producen.

Por otro lado, Luna (2001) manifiesta que la agricultura colombiana se ha visto afectada por las disminuciones de la productividad en las zonas hortícolas, caracterizada por el aumento en los costos de producción, dependencia de insumos externos, viéndose reflejada en la disminución de la calidad de vida de los agricultores e irrecuperables pérdidas de tipo ambiental por el uso indiscriminado de productos químicos. En este sentido, en Colombia, el cultivo de lechuga revela una disminución en rendimiento de 7,7\% y un descenso en la producción del 40,1\% para el año 2010 (AGRONET, 2012). Bajo este contexto, la presente investigación buscó evaluar la productividad en el cultivo de lechuga (L. sativa L.) var. Batavia bajo condiciones de macrotúnel con tres niveles de fertilización.

\section{MATERIALES Y MÉTODOS}

La investigación se desarrolló en la vereda San Felipe, ubicada en la zona centro occidental del municipio de Pasto, en la parte baja del volcán Galeras, a $5 \mathrm{~km}$ de la cabecera municipal. Se encuentra a una altura de $2710 \mathrm{msnm}$, con una precipitación promedio anual de $840 \mathrm{~mm}$, y una temperatura promedio de $13^{\circ} \mathrm{C}$. 
Los suelos de esta zona corresponden a una consociación Vitric Haplustands, fase moderadamente inclinada, originados de cenizas volcánicas que yacen sobre tobas de ceniza y lapilli; son muy profundos y moderadamente profundos, bien drenados y de fertilidad alta y moderada (IGAC, 2004). Según el análisis físico y químico del suelo realizado, el suelo presentó un nivel alto de materia orgánica y una textura franco arcillosa, con niveles medios a altos para macronutrientes y contenidos bajos de micronutrientes.

Diseño y construcción del macrotúnel: Se seleccionó un área de $80 \mathrm{~m}^{2}$, de la cual $32 \mathrm{~m}^{2}$ se destinaron para la construcción del macrotúnel y $32 \mathrm{~m}^{2}$ para el lote testigo, cultivo a campo abierto. Las dimensiones fueron de $8 \mathrm{~m}$ de largo y $4 \mathrm{~m}$ de ancho para los dos sitios. Las dos divisiones anteriores fueron separadas por una franja de 16 $\mathrm{m}^{2}$ (8 $\mathrm{m}$ de largo y $2 \mathrm{~m}$ ancho).

Cubierta del macrotúnel: Al finalizar la construcción de la estructura, se cubrió con plástico para invernadero calibre 8 de $9 \mathrm{~m}$ de ancho y 9 $\mathrm{m}$ de largo. El plástico se aseguró a la estructura con grapas en los dos extremos de la estructura. En cada lado de la estructura se instalaron dos puertas de $1 \mathrm{~m}$ de ancho y 1,5 $\mathrm{m}$ de alto para manejar las condiciones ambientales del macro- túnel. Las dimensiones finales del macrotúnel fueron de $2 \mathrm{~m}$ de alto en el centro, $4 \mathrm{~m}$ de ancho y $8 \mathrm{~m}$ de largo (Fig. 1).

Análisis de suelo. Para la investigación se realizó un análisis de suelos inicial, donde se obtuvieron los siguientes resultados: $\mathrm{pH}: 4,9$; M.O.S:11,5\%; P:31,5mg/kg; CIC: 24, Ca:4,57, Mg:1,52, K:0,72, Al:1,76 cmolc/kg. La textura del suelo fue franco arcillosa, el valor de la densidad del suelo fue de $0,80 \mathrm{~kg} / \mathrm{dm}^{3}$ a partir de estos resultados se realizó plan de fertilización utilizado en la presente investigación.

\section{Labores culturales}

Preparación del suelo: Se prepararon tres camas en el interior del macrotúnel y tres camas en campo abierto, con las siguientes dimensiones: $1 \mathrm{~m}$ de ancho, $8 \mathrm{~m}$ de largo y $0,20 \mathrm{~m}$ de altura.

Siembra y trasplante. Se utilizaron en total 288 plántulas de lechuga variedad Batavia; 144 plántulas en el cultivo bajo condiciones de macrotúnel y 144 plántulas en el testigo cultivo a campo abierto. En cada una de las tres camas se trazaron tres líneas donde se trasplantaron 16 plántulas en cada línea. La distancia entre líneas
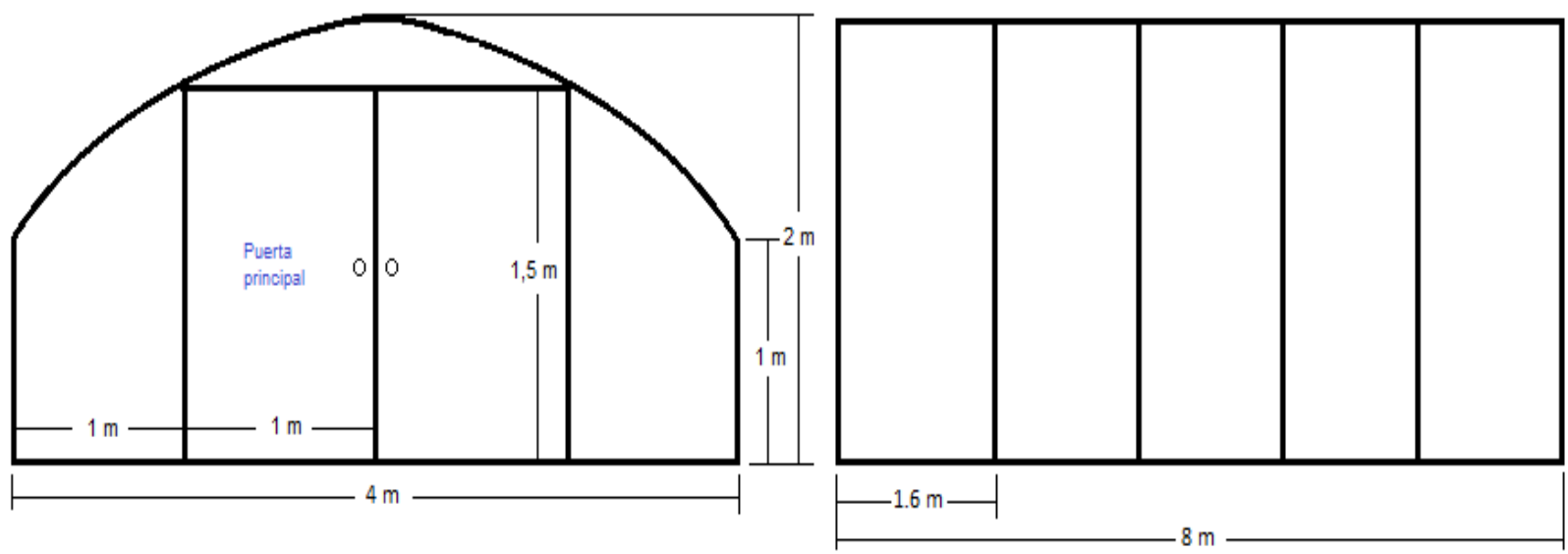

Figura 1. Planos de la estructura del macrotúnel 
fue de $35 \mathrm{~cm}$ y entre plantas $40 \mathrm{~cm}$. Se utilizaron 12 plantas por unidad experimental. Las plántulas que se llevaron al sitio definitivo tenían 30 días de sembradas en bandejas de germinación.

Manejo agronómico del cultivo. Se realizó el control manual de malezas, eliminando las que se encontraron cerca de la planta. En cuanto a plagas y enfermedades se realizó un manejo integrado, teniendo en cuenta que la medida de control no superó el costo del daño económico.

Se instaló un sistema de riego por goteo con 12 cintas de riego de 1 pulgada de diámetro (dos por cada cama) insertadas con conectores independientes a una tubería de 2 pulgadas de diámetro. Durante todo el ciclo del cultivo se utilizaron alrededor de $400 \mathrm{~mm}$ de agua ICA (1996).

Registro de las condiciones ambientales. Las lecturas de temperatura, humedad relativa y concentración de $\mathrm{CO}_{2}$ se registraron con el sensor de concentración de $\mathrm{CO}_{2}$ (Ref:CO200 Tecnología NDIR Infrarrojo no dispersivo); los datos se tomaron semanalmente en el interior y en el exterior del macrotúnel. La resistencia mecánica de las capas superficiales del suelo se registró con el penetrómetro (DIK-5561, Daiki rikakogyo Co., Ltd. Tokio, Japón).

\section{Variables de respuesta del cultivo}

Peso de la lechuga (PL). Se tomaron los pesos individuales de 10 plantas de lechuga por cada tratamiento, cuando más del $50 \%$ de las plantas de lechuga se encontraron en el punto de madurez de cosecha. Para lo anterior, se utilizó una balanza en el lugar de la investigación.

Días a cosecha (Dcos). Se contaron los días que tardaron las plantas en los seis tratamientos (dos coberturas y tres niveles de fertilización por cobertura) en llegar a la formación de la cabeza compacta, para lo cual se llevó un registro de la fecha del día del trasplante hasta la fecha del día de la cosecha, donde se colectó el material de la parcela útil para los análisis de laboratorio y la respectiva cosecha.

Diámetro de la cabeza (cm) (DC). Se tomó la medida de la longitud del plano ecuatorial con una cinta métrica, cuando se formó la cabeza compacta en más del 50\% del cultivo en los dos sistemas de cultivo (campo abierto y macrotunel), posteriormente, se calculó el diámetro con la siguiente fórmula:

$$
\begin{aligned}
& \mathrm{D}=\mathrm{C} / \Pi \\
& \mathrm{D}=\text { Diámetro de la cabeza } \\
& \mathrm{C}=\text { Longitud de la circunferencia }
\end{aligned}
$$

Porcentaje de materia seca de la parte aérea (\%MSA) y raíz (\%MSR). De las 12 plantas de lechuga que constituyeron área útil para cada tratamiento, se tomaron dos plantas de lechuga completas (parte aérea y raíz) cuando se formó la cabeza compacta, para los respectivos análisis de laboratorio. La selección se realizó al azar y éstas se llevaron en bolsas de papel al laboratorio de Fisiología Vegetal de la Universidad de Nariño. Se tomó su peso de materia fresca y luego se llevó a secar a $65^{\circ} \mathrm{C}$ por 72 horas; posteriormente se pesaron para obtener el peso de materia seca.

Rendimiento (R). Se pesaron las 12 unidades experimentales en el momento de la formación de la cabeza en el lugar de la investigación, los valores de peso se tomaron con una balanza electrónica LEXUS (0,01 de precisión), para posteriormente calcular el rendimiento $(\mathrm{kg} /$ $\mathrm{m}^{2}$ ), dividiendo el peso de las 12 unidades experimentales $(\mathrm{kg})$ entre el área útil de cada tratamiento $\left(1,6 \mathrm{~m}^{2}\right)$. 


\section{Diseño experimental}

Se utilizó un diseño experimental de bloques completos al azar (BCA) en un arreglo de franjas divididas con 6 tratamientos y tres repeticiones. Las dos franjas principales se llamaron "Ambientes" y se establecieron por las características de macrotúnel y el cultivo a campo abierto, la sub-franja correspondió a los niveles de fertilización N,P,K Alto $(115,69,210 \mathrm{~kg} / \mathrm{ha}), \mathrm{N}, \mathrm{P}, \mathrm{K}$, Medio (69, 23, 150 kg/ha) y N,P,K, Bajo $(46,11,5$, $90 \mathrm{~kg} / \mathrm{ha}$ ) (Tab.1).

Análisis de variables de respuesta. Las variables de respuesta se sometieron al Análisis de Varian$\mathrm{za}$, para aquellas variables que presentaron diferencias estadísticas significativas se les realizó la Prueba de Comparaciones Múltiples de Medias por medio del test de Tukey $(\mathrm{P}<0,05)$.

\section{Análisis económico}

Se utilizó la metodología propuesta por Perrin et al. (1976), para determinar cuál de los tratamientos genera las mejores condiciones econó- micas para lo cual se realizó un análisis económico comparativo de rendimientos y costos de producción (t/ha) para cada tratamiento.

\section{RESULTADOS Y DISCUSIÓN}

En los resultados presentados a continuación, es conveniente aclarar que en todo el desarrollo estadístico se denominó ambiente a los dos sistemas de cultivo evaluados (macrotunel y campo abierto), que fueron los ambientes sobre los cuales se aplicó los tres niveles de fertilización (alto, medio, bajo), sobre el cultivo de lechuga, en ese sentido los resultados obtenidos por variable de respuesta fueron los siguientes:

\section{Días a cosecha (Dcos)}

En esta variable se presentaron diferencias estadísticas al nivel de $(\mathrm{P}<0,05$ y $\mathrm{P}<0,01)$ para la variable ambientes (macrotunel y campo abierto), en cuatro de las cinco variables de cultivo evaluadas (Tab. 2).

Tabla 1. Distribución de los tratamientos con dos condiciones de cultivo (macrotúnel-campo abierto) y tres niveles de fertilización en forma de $\mathrm{N}_{1} \mathrm{P}_{2} \mathrm{O}_{5^{\prime}} \mathrm{K}_{2} \mathrm{O}$

\begin{tabular}{c|c|l|l}
\hline Franja principal & Tratamientos & $\begin{array}{c}\text { Ambientes } \\
\text { Fa. Principal }\end{array}$ & Nivel de Fertilización (Sub-franja) \\
\hline Franja principal & 1 & Macrotúnel & Alto $(115,69,210 \mathrm{de} \mathrm{N}, \mathrm{P}, \mathrm{K}, \mathrm{kg} / \mathrm{ha})^{* *}$ \\
$\left(32 \mathrm{~m}^{2}\right)$ & 2 & Macrotúnel & Medio $(69,23,150 \mathrm{de} \mathrm{N}, \mathrm{P}, \mathrm{K}, \mathrm{kg} / \mathrm{ha})^{*}$ \\
& 3 & Macrotúnel & Bajo $(46,11.5,90 \mathrm{de} \mathrm{N}, \mathrm{P}, \mathrm{K}, \mathrm{kg} / \mathrm{ha})^{* *}$ \\
\hline Franja principal & 4 & Campo abierto & Alto $(115,69,210 \mathrm{de} \mathrm{N}, \mathrm{P}, \mathrm{K}, \mathrm{kg} / \mathrm{ha})$ \\
$\left(32 \mathrm{~m}^{2}\right)$ & 5 & Campo abierto & Medio $(69,23,150 \mathrm{de} \mathrm{N}, \mathrm{P}, \mathrm{K}, \mathrm{kg} / \mathrm{ha})^{*}$ \\
& 6 & Campo abierto & Bajo $(46,11.5,90 \mathrm{de} \mathrm{N}, \mathrm{P}, \mathrm{K}, \mathrm{kg} / \mathrm{ha})$ \\
\hline
\end{tabular}

*Testigo, nivel de fertilización recomendado (Lara y Vallejo, 2010).

**Fertilizaciones tradicionales de la zona encontradas por el histórico de producción del agricultor 
Tabla 2. Cuadrado Medio de las variables de cultivo evaluadas en dos ambientes y tres niveles de fertilización

\begin{tabular}{l|r|r|r|r|r|r}
\hline Fuente de variación & \%MSA & \%MSR & \multicolumn{1}{c|}{ DC } & PL & \multicolumn{1}{c|}{ R } & \multicolumn{1}{c}{ Dcos } \\
\hline Modelo & 4,29 & 271,34 & 81,98 & 2,39 & 86,14 & 769,83 \\
Bloque & 0,36 & 81,51 & 3,69 & 0,04 & 1,34 & 1 \\
Fertilización & $0,78 \mathbf{n s}$ & $5,25 \mathbf{n s}$ & $5,47 \mathbf{n s}$ & $0,14 \mathbf{n s}$ & $5,18 \mathbf{n s}$ & $0,001 \mathbf{n s}$ \\
Bloque*Fertilización (Error a) & 1,05 & 53,88 & 15,53 & 0,07 & 2,36 & 4 \\
Ambientes & $0,04 \mathbf{n s}$ & $106,09 *$ & $48,02 *$ & $1,88 *$ & $67,74 *$ & $760,50 * *$ \\
Bloque*Ambientes (Error b) & 1,45 & 0,36 & 2,72 & 0,11 & 4,04 & 4,33 \\
Fertilización*Ambientes & $0,62 \mathbf{n s}$ & $24,25 \mathbf{n s}$ & $6,54 \mathbf{n s}$ & $0,15 \mathbf{n s}$ & $5,47 \mathbf{~ n s}$ & $0,001 \mathbf{~ n s}$ \\
Error residual & 1,12 & 10,44 & 3.26 & 0,11 & 3,79 & 2,67 \\
Total & 5,41 & 281,78 & 85,24 & 2,50 & 89,92 & 772,50 \\
Coef. Variación & 10,5 & 12,3 & 4,8 & 8,4 & 8,4 & 1,01 \\
\hline
\end{tabular}

* Diferencia significativa $(\mathrm{p}<=0,05),{ }^{* *}$ Diferencia altamente significativa $(\mathrm{p}<=0,01)$,

*** Diferencia altamente significativa $(\mathrm{p}<=0,001), \mathrm{ns}-$ No significativo

La Prueba de Comparación de Medias de Tukey (Tab. 3), indica que la mayor precocidad se alcanza en el cultivo bajo cubierta debido a que las plantas presentaron madurez de cosecha a los 74 días después del trasplante, a diferencia de los 87 días en el cultivo a campo abierto. Los resultados concuerdan con diferentes autores quiénes aseguran que el cultivo de lechuga bajo cubierta reduce el tiempo de cosecha en 12 días, lo que permite obtener hasta 5 cosechas en un año, una más en comparación con el cultivo a campo abierto.

Al respecto Hunter (2010) menciona que los macrotuneles se han utilizado para aumentar efectivamente los periodos de cosechas en el año para numerosos cultivos. Además, una ventaja de los cultivos que se obtienen fuera de temporada, es que demandan un incremento de precio cuando se venden en los mercados directos (Foord, 2004).

Igualmente Randin et al. (1999) señalan que el cultivo protegido tiene la capacidad de disminuir la fase de crecimiento en plantas de lechuga,lo cual se debe probablemente a la acumulación de calor en el interior del macrotúnel, donde se registra una temperatura promedio de $25,9^{\circ} \mathrm{C}$ en el día, $9,5^{\circ} \mathrm{C}$ más que en el cultivo a campo abierto $16,4^{\circ} \mathrm{C}$ (Tab. 4). Por su parte, Degiovanni et al. (2010) mencionan que en arroz, el crecimiento vegetal sigue la ley de Van'tHoff cuando la temperatura se encuentra entre los $10^{\circ} \mathrm{C}$ y los $30^{\circ} \mathrm{C}$.

Tabla 3.Valores promedios de las variables de cultivo en dos ambientes

\begin{tabular}{l|c|c|c|c|c|c}
\hline Parcela Principal & $\begin{array}{c}\text { Dcos } \\
\text { (Días) }\end{array}$ & $\begin{array}{c}\text { \%MSA } \\
\mathbf{( \% )}\end{array}$ & $\begin{array}{c}\text { \%MSR } \\
\mathbf{( \% )}\end{array}$ & $\begin{array}{c}\text { DC } \\
\mathbf{( c m )}\end{array}$ & $\begin{array}{c}\text { PL } \\
\mathbf{( k g )}\end{array}$ & $\begin{array}{c}\mathbf{R} \\
\mathbf{( k g / \mathbf { m } ^ { 2 } )}\end{array}$ \\
\hline Macrotúnel & $74,33 \mathrm{~b}$ & $5,27 \mathrm{a}$ & $15,66 \mathrm{a}$ & $20,33 \mathrm{a}$ & $2,24 \mathrm{a}$ & $13,46 \mathrm{a}$ \\
Campo abierto & $87,89 \mathrm{a}$ & $5,17 \mathrm{a}$ & $10,53 \mathrm{~b}$ & $17,07 \mathrm{~b}$ & $1,59 \mathrm{~b}$ & $9,56 \mathrm{~b}$ \\
\hline
\end{tabular}

Letras distintas indican diferencias significativas a $(\mathrm{P}<=0,05)$ 
Tabla 4. Promedio de temperatura $\left({ }^{\circ} \mathrm{C}\right)$, humedad relativa (\%HR) y concentración de $\mathrm{CO}_{2}(\mathrm{ppm})$ para el microclima del macrotúnel y las condiciones de campo abierto

\begin{tabular}{c|l|c|c|c}
\hline Tiempo de registro & Condiciones & $\begin{array}{c}\text { Temperatura } \\
\left({ }^{\circ} \mathbf{C}\right)\end{array}$ & $\begin{array}{c}\text { Humedad } \\
\text { Relativa (\%HR) }\end{array}$ & $\begin{array}{c}\text { Concentración } \\
\mathbf{d e ~ C O}_{2}(\mathbf{p p m})\end{array}$ \\
\hline \multirow{2}{*}{ Promedio día } & Campo abierto & 16,4 & 72 & 436 \\
\cline { 2 - 5 } & Macrotúnel & 25,9 & 55 & 423 \\
\hline \multirow{2}{*}{ Promedio noche } & Campo abierto & 10,3 & 91 & 471 \\
\cline { 2 - 5 } & Macrotúnel & 10,9 & 89 & 466 \\
\hline
\end{tabular}

Por otro lado, no se encontraron diferencias estadísticas para la variable días a cosecha en los tratamientos de niveles de fertilización, ni para la interacción del ambiente dosis de fertilización (Tab. 2), por lo tanto, es posible que la fertilización por el corto tiempo de evaluación (74 y 83 días para macrotúnel y campo abierto, respectivamente), posiblemente no se haya manifestado en la precocidad del cultivo de lechuga, además que dentro del ensayo no se contempló un testigo absoluto (sin fertilización), que hubiese dado una opción más clara en este aspecto. Al respecto Chimenti (1999) evaluó el efecto de la fertilización orgánica comparada con una mixta y convencional, en tres variedades de lechuga bajo invernadero y no manifiesta respuesta en precocidad por efecto de la fertilización, aunque si por el tipo de variedad cultivada.

\section{Materia seca aérea (\%MSA) y raíz (\%MSR)}

En la Tabla 2, se indica que para el porcentaje de materia seca de la parte aérea, no se encontraron diferencias estadísticas significativas para el cultivo de lechuga en los dos tipos de ambiente ni para los niveles de fertilización. El promedio del porcentaje de materia seca de la parte aérea fue de $5,27 \%$ bajo cualquiera de los seis tratamientos propuestos. Al respecto, Alzate y Loaiza (2010) reportan que el porcentaje de agua en las plantas de lechuga es alrededor de $94 \%$.
Igualmente dentro de las observaciones realizadas en el transcurso del desarrollo del cultivo, se determinó que bajo las condiciones del macrotúnel, se produjo un mayor crecimiento de la hoja expandiendo la lámina foliar, observándose al cultivo con una mayor cantidad de hojas. En este sentido, Santos et al.(2009) sustentan que desde los 28 días después del trasplante, las plantas de lechuga aumentan un $54,2 \%$ su área foliar y desde los 14 días después del trasplante, incrementan un $64,18 \%$ en el número de hojas, en plantas evaluadas bajo condiciones protegidas, en comparación con el cultivo a campo abierto. En cuanto a la materia seca de raíz en condiciones de macrotúnel, alcanzó un promedio 15,66\% mientras que en el cultivo a campo abierto se obtuvo un $10,53 \%$ (Tab. 3).

\section{Diámetro de cabeza (DC)}

Para la variable de diámetro de cabeza, en el Análisis de Varianza (Tab. 2), se observa diferencias significativas en cuanto al cultivo de lechuga en los dos tipos de ambiente (macrotúnel- campo abierto). La Prueba de Comparación de Medias de Tukey (Tab. 3), revela que el mayor diámetro de cabeza se obtuvo en el cultivo de lechuga bajo condiciones de macrotúnel con 20,33 cm en comparación con el cultivo a campo abierto con $17,07 \mathrm{~cm}$. Al respecto Domínguez (2002), manifiesta que la lechuga bajo cubierta en microtúneles presenta un diámetro de cabeza $11 \%$ mayor comparado con el testigo a campo abierto. 
El incremento del diámetro de cabeza en 16\%, en el cultivo bajo cubierta, puede posiblemente estar vinculado a la disminución de la humedad relativa del interior del macrotúnel donde se registró un promedio de $55 \%$ en comparación con el testigo a campo abierto $72 \%$. Al respecto, se puede manifestar quela humedad del aire está relacionada con la velocidad de transpiración de la planta y que ante una elevada humedad relativa la planta transpira poco lo que reduce el transporte de nutrientes desde las raíces hacia las hojas.

Por otra parte, es importante destacar que la temperatura es un factor clave para la formación y calidad de la cabeza de la planta de lechuga, estudios realizados por Segovia et al. (1997) demuestran que la temperatura provoca una influencia directa en los órganos de esta especie. Debido a lo anterior, durante la investigación, las puertas de la estructura del macrotúnel se abrieron diariamente, al medio día en días soleados y al finalizar la tarde, desde el momento del trasplante hasta el final del ensayo en un total de 90 días, tiempo total de duración del experimento en los dos ambientes.

Respecto a los niveles de abonamiento no se encontraron diferencias estadísticas para los tres niveles evaluados, ni para la interacción de ambiente por niveles de fertilización (Tab. 2). Los resultados indican que la menor dosis de fertilización (46, 11.5, 90 de N,P,K, kg/ ha) es suficiente para alcanzar valores iguales estadísticamente en diámetro de cabeza para los niveles de fertilización, resultado importante en la zona por los costos en la producción que representa este renglón en el compendio de los costos del cultivo, en promedio el diámetro de cabeza fue mayor en el macrotúnel $(20 \mathrm{~cm})$ que en campo abierto $(17 \mathrm{~cm})$.

\section{Peso de la lechuga (PL)}

En el Análisis de Varianza en la (Tab. 2), se puede observar que para la variable peso de lechuga se encontraron diferencias significativas en cuanto al cultivo de lechuga en los dos tipos de ambiente. La Prueba de Comparación de Medias de Tukey (Tab. 3) establece que el mayor peso de la lechuga se obtuvo en el cultivo bajo condiciones de macrotúnel con un promedio de 2,24 $\mathrm{kg} /$ planta en comparación con el testigo (cultivo a campo abierto) con un promedio de 1,59 $\mathrm{kg} /$ planta, siendo el incremento en el peso de la lechuga de 40,88\%. Resultados similares son reportados por Rader y Karlsson (2006) quienes manifiestan que la lechuga cultivada en macrotúnel incrementa en un $49,23 \%$ de su peso en comparación con el cultivo a campo abierto. Al respecto, la acumulación de calor en el interior de un invernadero promueve el calentamiento de la zona radicular y como consecuencia se incrementa la acumulación de biomasa y la absorción de nutrientes de la lechuga (Economakis, 1997; Moorby y Graves (1980).

No se encontraron diferencias entre los niveles de fertilización, ni para la interacción del ambiente por las dosis de fertilización. Los resultados indican que se puede obtener plantas de lechuga con pesos estadísticamente iguales con la menor dosis de fertilización (46, 11.5, 90 de $\mathrm{N}, \mathrm{P}, \mathrm{K}, \mathrm{kg} / \mathrm{ha}$ ) en cualquiera de los dos ambientes, macrotúnel y campo abierto, posiblemente por haber evaluado un solo ciclo de cultivo y la no colocación de un testigo absoluto (tratamiento sin fertilización). Los valores de biomasa en las plantas de lechuga cultivadas en condiciones de macrotúnel puede ser provocado por la mayor asimilación y utilización de los nutrientes (Santos et al., 2009) mejorando la eficiencia de los fertilizantes químicos y aminorando la dependencia de los mismos. 
Los resultados de éste ensayo, en que el cultivo de lechuga a campo abierto requiere bajos niveles de fósforo $28 \mathrm{~kg} /$ ha de $\mathrm{P}_{2} \mathrm{O}_{5^{\prime}}$ mas difieren en dosis de Nitrógeno $45 \mathrm{~kg} / \mathrm{ha}$ y potasio $208 \mathrm{~kg} / \mathrm{ha}$ de $\mathrm{K}_{2} \mathrm{O}$ para una producción de $40 \mathrm{t} / \mathrm{ha}$, situación que concuerda con resultados obtenidos por autores como Castro (1998), Guerrero (1998) que encontraron valores similares a los de la presente investigación.

\section{Rendimiento (R)}

Al realizar el Análisis de Varianza (Tab. 2) se presentaron diferencias estadísticas significativas en cuanto al cultivo de lechuga bajo condiciones de macrotúnel y a campo abierto. La Prueba de Comparación de Medias de Tukey (Tab. 3), revela que el mayor rendimiento se obtuvo en el cultivo de lechuga bajo condiciones de macrotúnel con $13,46 \mathrm{~kg} / \mathrm{m}^{2}$ en contraste con el cultivo a campo abierto con $9,56 \mathrm{~kg} / \mathrm{m}^{2}$, de tal manera que se obtuvo un incremento de $41 \%$ en rendimiento. Al respecto Goto et al. (2000) manifiestan que es posible mayor acumulación de materia seca en lechuga bajo sistemas forzados. Por su parte Shedlosky y White (1987) afirman que al interior del macrotúnel se ha demostrado que el calentamiento de la zona radicular compensa el efecto perjudicial de las temperaturas de aire frío $\left(<16^{\circ} \mathrm{C}\right)$ para las plantas de invernadero favoreciendo los procesos, situación que no contribuyó con los niveles de fertilización ya que no hubo diferencias estadísticas significativas, para los niveles de fertilización ni para la interacción del ambiente por los niveles de fertilización (Tab. 2) de tal manera que se obtuvieron rendimientos estadísticamente iguales en el cultivo de lechuga con los tres niveles de fertilización alto $(115,69,210)$, medio $(69,23,150)$, bajo $(46,11.5$, 90) kg/ha de N, P, K.

Los valores de rendimiento observados, no concuerdan con los reportados por Lara y Vallejo (2010), quienes manifiestan que con la aplicación de $60,23,150 \mathrm{~kg} / \mathrm{ha}$ de $\mathrm{N}, \mathrm{P}, \mathrm{K}$, se obtiene un rendimiento estadísticamente superior a la dosis baja 46, 11.5, $90 \mathrm{~kg} / \mathrm{ha}$ de N, P, K. Es posible que el efecto de la fertilización en condiciones de campo abierto sea inhibido por problemas de orden físico del suelo, donde se observó presencia de encostramiento superficial con valores de $0,41 \mathrm{MPa}$ en macrotunel y de $1,45 \mathrm{MPa}$ en campo abierto con humedad gravimétrica del 18\%. El valor observado a campo abierto posiblemente fue un factor negativo en la entrada de agua en el suelo ya que la costra superficial estaba en el límite superior de compactación lo cual puede haber afectado los procesos nutricionales (Amézquita, 1994; Amézquita et al., 2002).

\section{Análisis Económico}

El análisis económico fue realizado para dos ambientes con la menor dosis de fertilización $(46,11,5,90$ de N, P, K, ) y para calcular la amortización de la estructura del macrotúnel se estimó una vida útil de 4 años (Tab. 5).

Los resultados indican que se alcanzaron mayores utilidades para el primer año con el cultivo de lechuga bajo cubierta en macrotúnel, con \$ 32620 por cada $\mathrm{m}^{2}$ en comparación con el cultivo a campo abierto donde se presentaron utilidades de 7,974 pesos por cada $\mathrm{m}^{2}$ (Tab. 6).

Es importante resaltar, que los dos sistemas de cultivo presentan excelentes rentabilidades. La mayor rentabilidad se genera en el sistema de cultivo bajo condiciones de macrotúnel (605\%) en comparación con la producción de lechuga a campo abierto (588\%); además, la lechuga que proviene de condiciones protegidas se caracteriza por tener mayor aceptación en el mercado, mejores precios y se puede obtener el producto en un periodo corto de tiempo. Además, la inversión que se realiza en la siembra y el mantenimiento del cultivo, se encuentra protegida de pérdidas causadas por las adversidades del clima. 
Tabla 5. Costos de producción de lechuga cultivada a campo abierto y en condiciones de macrotúnel

\begin{tabular}{lcc}
\hline Costos de Producción & $\begin{array}{c}\text { Campo } \\
\text { abierto } \\
\text { (Pesos) }\end{array}$ & $\begin{array}{c}\text { Macrotúnel } \\
\text { (Pesos) }\end{array}$ \\
\hline Labores & 3000 & 3000 \\
Preparación semillero & 3900 & 3900 \\
Trasporte fertilizante & 10000 & 10000 \\
Trasporte cosecha & 12000 & 12000 \\
Implementación sistema de riego & $\mathbf{2 8 9 0 0}$ & $\mathbf{2 8 9 0 0}$ \\
\hline Total labores & & \\
\hline Insumos & 3000 & 3000 \\
Fertilizante & 2000 & 2000 \\
Semilla & $\mathbf{5 0 0 0}$ & $\mathbf{5 0 0 0}$ \\
\hline Total insumos & & \\
\hline Sistema de Riego & 30000 & 30000 \\
Cinta de goteo & 24000 & 24000 \\
Conectores & 30000 & 30000 \\
Tubería principal & $\mathbf{8 4 0 0 0}$ & $\mathbf{8 4 0 0 0}$ \\
Total sistema de Riego & $\mathbf{1 0 5 0 0}$ & $\mathbf{8 4 0 0}$ \\
\hline Amortización 2 Años & & \\
\hline Macrotúnel & & 120000 \\
Varas de Bambú & & 180000 \\
Plástico calibre 8 & & 10000 \\
Grapas & & 4000 \\
Bisagras & & 30000 \\
Codos de hierro & $\mathbf{3 7 6 0 0 0}$ \\
Guaduas & & $\mathbf{1 8 8 0 0}$ \\
Total Macrotúnel & $\mathbf{6 1 1 0 0}$ \\
Amortización 4 años & & $\mathbf{2 4 4 4 0 0}$ \\
\hline COSTOS TOTAL POR CICLO & & \\
\hline COSTO TOTAL POR AÑO & & \\
\hline & & \\
& & \\
\hline
\end{tabular}

Los costos se realizaron con base en el área de la franja principal $\left(32 \mathrm{~m}^{2}\right)$

SMMLV/2014 \$616.500 MCT

\section{CONCLUSIONES}

El cultivo bajo ambiente de macrotúnel, mostró ser más eficiente en cuanto al desarrollo del cultivo y presentó mayores valores en cuanto a las variables productivas de lechuga.

El rendimiento bajo cobertura plástica fue superior en $41 \%$, respecto al ambiente campo abierto.

La producción de lechuga en el sistema de macrotúnel, mostró ser más rentable que a campo abierto en un 507\% en la zona evaluada.

No se observó influencia de la interacción fertilización y ambiente en las diferentes variables de cultivo evaluadas.

\section{BIBLIOGRAFÍA}

AGRONET, 2012. Estadísticas agropecuarias. En: Agronet, http://www.agronet.gov.co/ www/htm3b/ReportesAjax/VerReporte.aspx; consulta: enero, 2013.

ALPI, A. y TOGNONI, F. 2010. Cultivo en invernadero. Actual orientación técnica y científica. 3 ed. Madrid, Mundi-prensa. 13 - 78p.

ALZATE, J.y LOAIZA, L. 2010. Monografía del cultivo de la lechuga. En: Inteligencia en agroproducción. Primera edición. Colinagro, Bogotá, 2-4 p.

Tabla 6. Análisis económico de la producción de lechuga a campo abierto y bajo condiciones de macrotúnel

\begin{tabular}{lcccccc}
\hline $\begin{array}{c}\text { Condiciones } \\
\text { del cultivo }\end{array}$ & $\begin{array}{c}\text { Rendimiento } \\
\left(\mathbf{k g} / \mathbf{m}^{\mathbf{2}}\right)\end{array}$ & $\begin{array}{c}\text { Rendimiento } \\
\left(\mathbf{k g} / \mathbf{m}^{2} / \mathbf{a n ̃ o}\right)\end{array}$ & $\begin{array}{c}\text { Ingresos } \\
\mathbf{1 0 0 0} \mathbf{p e s o s} / \\
\mathbf{k g} / \mathbf{a n ̃ o}\end{array}$ & $\begin{array}{c}\text { Costos } \\
\left(\mathbf{p e s o s} / \mathbf{m}^{\mathbf{2}}\right)\end{array}$ & $\begin{array}{c}\text { Utilidades } \\
(\mathbf{p e s o s} / \\
\left.\mathbf{m}^{2} / \mathbf{a n ̃ o}\right)\end{array}$ & $\begin{array}{c}\text { Rentabilidad } \\
\mathbf{( \% )}\end{array}$ \\
\hline Campo abierto & 9,56 & 38,24 & 38240 & 5550 & 32620 & 588 \\
Macrotúnel & 13,46 & 53,84 & 53840 & 7637 & 46203 & 605 \\
\hline
\end{tabular}


AMÉZQUITA, E. 1994. Las propiedades físicas y el manejo productivo de los suelos. En: Silva, F (ed.). Fertilidad de suelos, diagnóstico y control. Sociedad Colombiana de ciencias del Suelo. Santa Fé de Bogotá, Colombia. 137 - 154.

AMÉZQUITA, E., MADERO, E., JARAMILLO, R. y VIVEROS, R. 2002. Impacto físico de la intervención agrícola sobre un Vertisol del Valle del Cauca Colombia, Boletín de Suelos. 35p.

CASTILLA, N. 2007. Invernaderos de plástico. Tecnología y manejo. 2 ed. Madrid: Mundiprensa. $25-252 p$

CASTRO, H. 1998. IV Seminario sobre Hortalizas: Producción y fertilización de hortalizas en Colombia. 195-219 p.

CHIMENTI, S. C. 1999. Evaluación del efecto de una fertilización orgánica comparada con una mixta y convencional, en tres variedades de lechuga (Lactuca sativa L.) bajo invernadero. Tesis Ingeniero Agrónomo, Facultad de Ciencias Silvoagropecuarias, Universidad Mayor, Santiago, Chile.

DEGIOVANNI, V., MARTINEZ, C y MOTTA, F. 2010. Producción Eco-eficiente del arroz en América Latina. Tomo 1. Centro Internacional de Agricultura Tropical. CIAT. 1-24 p.

DOMÍNGUEZ, M. 2002. Evaluación de la producción de lechuga (LactucasativaL.) en época lluviosa bajo condiciones protegidas (microtúneles) en La Esperanza, Intibucá, Honduras. En: Informe técnico. Fundación Hondureña de Investigación Agrícola FHIA, proyecto la Esperanza. Lima, Cortes-Honduras. $15-20 \mathrm{p}$.

ECONOMAKIS, C. D. 1997. Effect of rootzone temperature on growth and water uptake by lettuce plants in solution culture. Acta Horticulturae. 449(1):199-203.

FOORD, K. 2004. High tunnel marketing and economics. Regents of the Univ.Of Minnesota. The Minnesotuniversity, In: http://www. extension.umn.edu/distribution/horti culture/ components; consulta: noviembre, 2013.

GUERRERO, R. 1998. Fertilización De Cultivos En Clima Frío. Monomeros Colombo Venezolanos. S.A. (E.M.A), Segunda edición. Impresión Saenz y Cia. Ltda. Santa Fe de Bogotá. Colombia. 425p.

GOTO, R., M.M. ECHER, V.F., GUIMARÃES, A.G., CARNEIRO J., R.B.F. BRANCO a y J.D. RODRIGUES.2000. Growth and production of three lettuce cultivars under protected and open filed conditions. Revista Horticultura Brasileira. 20(2):1-4 p.

HUNTER, B. L. 2010. “Enhancing Out-of-Season Production of Tomatoes and Lettuce Using High Tunnels". All Graduate Theses and Dissertations. Paper. 811p.

INSTITUTO GEOGRÁFICO AGUSTÍN CODAZZI (IGAC). 2004. Estudio general de suelos y zonificación de tierras. Departamento de Nariño. Bogotá: IGAC. CD-ROM.

INSTITUTO COLOMBIANO AGROPECUARIO (ICA). 2006.En: Primer Curso Nacional de Hortalizas. Conferencias/El instituto. Mosquera. Bogota: ICA,1996. 285p.

JENSEN, M.H.2000.Plasticulture in the global community: View of the past and future. Proc.15th Intl. Congr. Plastics Agr., 29th Natl. Agr. Plastics Congr. Appendix A: 1-11 
LAMONT, W. 2009. Overview of the Use of High Tunnels Worldwide. En: Hortitechnology. Department of Horticulture, Pennsylvania State University, 206 Tyson Bldg., University Park, PA 16802. 19(1).

LARA, C. y VALLEJO, R. 2010. Respuesta de la lechuga (Lactuca sativa L.) var. Batavia a la aplicación de fuentes simples de N-P-K en dos zonas del altiplano de Pasto. Trabajo de Grado, Ingeniero Agrónomo. Facultad de Ciencias Agrícolas. Universidad de Nariño. Pasto. 20p.

LUNA, L. 2001. Producción, uso y manejo de bioestimulantes, abonos orgánicos, acondicionadores y biofertilizantes a partir de fuentes no convencionales. Programa nacional de transferencia de tecnología agropecuaria, PRONATA, CORPOICA, Málaga, Santander. Colombia. 60p.

MALAVOLTA, E. 2006. Manual de nutrição mineral de plantas. São Paulo, Ed. Agronômica Ceres. 631p.

MOORBY, J. yC. J. GRAVES. 1980. Root and air temperature effects on growth and yield of tomatoes and lettuce. Acta Horticulturae. 98(1):29-44.

PERRIN, R.H., WINKELMANN, D., MOSCARDI, E. y ANDERSON, J.1976. Formulación de recomendaciones a partir de datos agronómicos. Un manual metodológico de evaluación económica. México, Centro Internacional de Mejoramiento de Maíz y Trigo CYMMYT. 54p.

RADER, B. y KARLSSON, M. 2006. Northern Field Production of Leaf and Romaine Lettuce using a High Tunnel.En: Hortechnology. Department of plant, Animal and Soil Sciences, University of Alaska, Fairbanks. 649- 654 p.
RANDIN, B., C. REISSER, J., R. MATZENAUER y H. BERGAMASCHI, 1999. Growth of lettuce cultivars conducted in cultivation protected and open Field. Horticultura Brasileira. 22(2):178-181.

SANTOS, F. B. G., LOBATO, R.B., SILVA, D., SCHIMIDT, COSTA., G.A.R. ALVESy OLIVEIRA, N. 2009. Growth of Lettuce (Lactuca Sativa L.)In Protected Cultivation and Open Field. Journal of Applied Sciences Research. 5(5):529 - 53.

SEGOVIA, J.F.O., J.L. ANDRIOLO., G.A. BURIOL y F.M. SCHNEIDER. 1997. Comparison of the growth and development of lettuce (Lactuca sativa L.) in interior and exterior of a polyethylene chamber in Santa Maria. Ciência Rural. 27(1):37-41 p.

SHEDLOSKY, M. E. y WHITE, J.W.1987. Growth of bedding plants in response to rootzone heating and night temperature regimes. J. Amer. American Society for Horticultural Science. 112(2):290-295 p.

WELLS, O.S. 2000. Season extension technology. Proc.15th Intl. Congr. Plastics Agr., 29th Natl. Agr. Plastics Congr. Appendix B: 1-7 p.

WELLS, O.S y J.B. LOY. 1993. Rowcovers and high tunnels enhance crop production in the northeastern United States. HortTechnology. 3:92 - $95 \mathrm{p}$. 